\title{
MÉTODO DE DETECÇÃO DO CÂNCER DE MAMA E SUAS IMPLICAÇÕES
}

Adriane Pires Batiston¹; Edson Mamoru Tamaki²; Mara Lisiane de Moraes dos Santos³; Luiza Helena de Oliveira

Cazola $^{4}$

RESUMO: O objetivo deste estudo foi conhecer o método de detecção e sua associação com o estadiamento do câncer de mama entre mulheres usuárias de um hospital público. Realizou-se um estudo transversal, no qual investigou-se as variáveis: idade, procedência, história familiar, estadiamento clínico e método de detecção. Para análise dos dados, utilizouse a estatística descritiva com nível de significância de 5\%. Estudou-se 223 mulheres com idade média de 51,3 \pm 12,41 anos, sendo 66,4\% residentes na capital e 33,6\% no interior do estado. A história familiar foi relatada por 24,5\% das mulheres. Em relação à forma de detecção observou-se as seguintes freqüências: auto-exame (76,7\%), exame clínico (15,7\%) e mamografia (7,6\%).O estadiamento clínico esteve associado ao método de detecção $(\leq 0,05)$ e ao local de residência $(\leq 0,05)$. A detecção do câncer de mama foi em maioria realizada pela própria mulher. O estadiamento clínico esteve associado ao método de detecção e à procedência.

PALAVRAS-CHAVE: Câncer de mama; Diagnóstico precoce; Epidemiologia.

\section{DETECTION METHOD OF THE BREAST CANCER AND ITS IMPLICATIONS}

\begin{abstract}
This study aimed to know the detection method and how it was associated with the breast neoplasms staging, among women users of a public hospital. A cross-sectional study was carried out in which the following key variables were researched: age, provenance, family history,, clinical staging and method for tumor detection. Descriptive statistics was used for data analysis, with significance level of 5\%. 223 women were analyzed, with the average age of 51,3 $\pm 12,41$ years, $66,4 \%$ of the patients lived in the capital city and 33,6\% in the countryside. The family history of breast cancer was reported by $24,5 \%$ of the users. In relation to the method for tumor detection, the subsequent frequencies were observed: breast self-examination (76,7\%), breast clinical examination (15,7\%) and mammography (7,6\%). Clinical staging have been significantly related with the method for tumor detection $(\leq 0,05)$ and to the place of living $(\leq 0,05)$. Breast cancer detection in the group analyzed was mostly done by the woman herself. Clinical staging was related to the method for tumor detection and also with the provenance.
\end{abstract}

KEYWORDS: Breast neoplasms; Early diagnosis; Epidemiology.

\section{MÉTODO DE DETECCIÓN DEL CÁNCER DE MAMAY SUS IMPLICACIONES}

RESUMEN: El objetivo del presente estudio fue conocer el método de detección y su asociación con la estadificación del neoplasias de la mama entre mujeres usuarias de un hospital público. Se realizó un estudio transversal, en el cual se investigaron las variables: edad, procedencia, historia familiar, estadificación clínica y el método de detección. Para análisis de los datos, se utilizó la estadística descriptiva con nivel de significancia del 5\%. Fueron estudiadas 223 mujeres con edad media de 51,3 + 12,41 años, siendo 66,4\% residentes de la capital y 33,6\% del interior del estado. La historia familiar fue relatada por 24,5\% de las mujeres. En relación a la forma de detección del cáncer se observaron las siguientes frecuencias: autoexamen (76,7\%), examen clínico (15,7\%) y mamografía (7,6\%). La estadificación clínica estuvo asociada al método de detección $(\leq 0,05)$ y al local de residencia $(\leq 0,05)$. La detección del cáncer de mama se realizó, mayormente, por la propia mujer. La estadificación clínica estuvo asociada al método de detección y a la procedencia.

PALABRAS CLAVE: Neoplasias de la mama; Diagnóstico Precoz, Epidemiología.

\footnotetext{
${ }^{1}$ Doutoranda no Programa Multiinstitucional de Pós-Graduação em Ciências da Saúde-UnB/UFG/UFMS. Professora do Departamento de Tecnologia de Alimentos e Saúde Pública da Universidade Federal de Mato Grosso do Sul-UFMS.

${ }^{2}$ Orientador no Programa Multiinstitucional de Pós-Graduação em Ciências da Saúde-UnB/UFG/UFMS. Professor do Departamento de Tecnologia de Alimentos e Saúde Pública da UFMS.

${ }^{3}$ Doutoranda no Programa Multiinstitucional de Pós-Graduação em Ciências da Saúde-UnB/UFG/UFMS. Professora do Departamento de Tecnologia de Alimentos e Saúde Pública da UFMS.

${ }^{4}$ Doutoranda do Programa de Pós-Graduação em Saúde e Desenvolvimento na Região Centro-Oeste-UFMS. Professora do Departamento de Tecnologia de Alimentos e Saúde Pública da UFMSul.
}

Autor correspondente:

Adriane Pires Batiston

Rua Robert Spenglei, 42 - 79004-070 - Campo Grande-MS

Recebido: 15/11/08

E-mail: apbatiston@hotmail.com

Aprovado: 17/03/09 


\section{INTRODUÇÃO}

Entre os diversos tipos de câncer, o de mama (CM) apresenta-se como um grave problema de saúde pública sendo o segundo tipo mais freqüente no mundo e o primeiro na população feminina. No Brasil, estimase que no ano de 2008, cerca de 49.400 mulheres receberão o diagnóstico do $\mathrm{CM}$, isto representa uma taxa bruta de 50,71 novos casos para cada 100.000 mulheres. O Estado de Mato Grosso do Sul, encontrase entre aqueles com maiores taxas de incidência da doença; em 2008 serão diagnosticados 560 novos casos de CM no estado ${ }^{(1)}$.

A despeito do elevado número de pesquisas já conduzidas sobre o câncer de mama, a sua etiologia ainda não está totalmente esclarecida, sendo a mesma atribuída a uma interação de fatores, que, de certa forma, são considerados determinantes no desenvolvimento da doença ${ }^{(2)}$.

O diagnóstico do $\mathrm{CM}$ precoce assume papel decisivo para um melhor prognóstico. Devido à sua etiopatogenia complexa e multifatorial, o CM não pode ser prevenido. O termo prevenção em epidemiologia é utilizado tanto para designar medidas capazes de impedir o aparecimento de um agravo à saúde (prevenção primária), como em sentido mais amplo, para medidas que impeçam o desenvolvimento do estágio seguinte das doenças (prevenção secundária) ${ }^{(3)}$. Desta forma, os esforços para melhoria dos indicadores do CM são direcionados na busca por medidas que antecipem seu diagnóstico, minimizando a agressividade do tratamento administrado e reduzindo as taxas de mortalidade, sendo estas medidas classificadas como prevenção secundária ${ }^{(4-5)}$.

Entre as mulheres brasileiras, aproximadamente $60 \%$ das neoplasias malignas da mama são diagnosticadas em estádios III e IV, situação inversa é observada em países como Estados Unidos e Inglaterra, nos quais $80 \%$ das mulheres recebem o diagnóstico do CM em estádios I e II ${ }^{(6-7)}$.

Nos últimos 40 anos, métodos capazes de diagnosticar precocemente o CM foram difundidos em todo o mundo. A busca por maior acurácia, na detecção de tumores iniciais da mama, estimulou a realização de importantes estudos que resultaram em estratégias nas quais estão pautados os programas de rastreamento e detecção precoce do $\mathrm{CM}^{(8)}$.

O objetivo primário dos programas de rastreamento é impactar as taxas de mortalidade pela doença, além de favorecer seu diagnóstico precoce e conseqüentemente poder dispor de mais opções terapêuticas, com menores efeitos indesejados nos âmbitos físico, mental e social ${ }^{(9)}$. Os programas de rastreamento e detecção precoce do CM, em sua maioria apóiam-se na tríade constituída pelo autoexame das mamas (AEM), o exame clínico das mamas (ECM) e pela mamografia ${ }^{(10-11)}$. A utilização destes três métodos tem sido amplamente discutida na literatura científica. Estudos buscam investigar o impacto de cada um deles principalmente no aumento da sobrevida das mulheres com $\mathrm{CM}^{(12-16)}$.

No Brasil, as recomendações do Instituto Nacional de Câncer (INCA) para rastreamento e detecção do CM são: 1. ECM em mulheres a partir dos 40 anos de idade; 2. ECM mais exame mamográfico bianual em mulheres de 50 a 69 anos de idade; e, 3 . ECM mais exame mamográfico anual em mulheres com risco elevado a partir dos 35 anos de idade ${ }^{(17)}$.

Pode-se considerar que embora a mamografia seja um método capaz de detectar o CM inicial, ainda não-palpável e, conseqüentemente, reduzir a mortalidade pela doença, em países como o Brasil, a redução no número de óbitos acaba não sendo observada, já que muitas mulheres não têm acesso a este procedimento como estratégia de rastreamento.

Diante do exposto, o objetivo deste estudo foi conhecer o método de detecção e sua associação com o estadiamento clínico do câncer de mama entre mulheres usuárias do ambulatório de fisioterapia do serviço de Oncologia de um hospital público.

\section{CASUÍSTICA E MÉTODO}

Trata-se de um estudo descritivo do tipo transversal realizado no Serviço de Oncologia de um hospital público na cidade de Campo Grande, Estado de Mato Grosso do Sul.

Foram incluídas neste estudo as 223 mulheres que utilizaram o serviço de fisioterapia entre os anos de 1998 e 2003.

Utilizou-se como fonte de dados as fichas de avaliação fisioterápica arquivadas no próprio setor de Fisioterapia e os prontuários das mulheres que receberam o diagnóstico de CM disponíveis no Serviço de Arquivo Médico e Estatística (SAME) do referido hospital. Fontes de dados como os prontuários tornaram-se muito utilizadas para estudos de morbidade, pois permitem o seguimento do paciente e conseqüentemente o acompanhamento de sua saúde, visto que as informações referentes às consultas, internações, exames complementares, tratamentos e medicamentos são documentadas nos prontuários ${ }^{(3)}$. 
Foram investigadas as seguintes variáveis: idade da mulher no momento do diagnóstico: expressa em anos completos; procedência expressa em: capital (mulheres residentes em Campo Grande, Mato Grosso do Sul (MS), interior (mulheres residentes em cidades do interior do Estado de MS); história familiar expressa em sim (parentes de primeiro e/ou segundo grau com história de CM) ou não; forma de detecção do tumor classificada em: auto-exame das mamas (exame realizado pela própria paciente), exame clínico das mamas (exame das mamas realizado por profissional de saúde habilitado), e mamografia; e estadiamento clínico: expresso em I, II, III e IV.

O estudo respeitou os critérios éticos conforme a Resolução do Conselho Nacional de Saúde n 196/96, sendo aprovado pelo Comitê de Ética em Pesquisa da Universidade Estadual de Campinas, parecer n 418/02.

Para análise dos dados utilizou-se a estatística descritiva e o teste não-paramétrico qui-quadrado quando aplicável com nível significância de 5\%.

\section{RESULTADOS}

Entre as 223 mulheres incluídas neste estudo, 148
(66,4\%) residem na cidade de Campo Grande, capital do Estado de Mato Grosso do Sul e 75 (33,6\%) são residentes em cidades do interior do Estado. No momento do diagnóstico, as mulheres apresentaram idade variando entre 22 a 85 anos, sendo a idade média de 51,3 $\pm 12,41$ anos (média, \pm desvio padrão). Entre as mulheres estudadas, 54 (24,5\%) relataram história familiar de CM.

Do total de casos de CM diagnosticados, 171 (76,7\%) foram detectados por meio do AEM, 35 (15,7\%) foram detectados durante o ECM e 17 (7,6\%) foram detectados por meio de exame mamográfico. A forma de detecção do CM não esteve relacionada com as variáveis: local de residência ( $p=0,29)$, idade da mulher $(p=0,80)$ e presença de antecedentes familiares de $\mathrm{CM}(\mathrm{p}=0,95)$. O estadiamento clínico não esteve relacionado com as variáveis: história familiar de CM $(p=0,24)$ e idade $(p=0,14)$. Houve relação significativa entre estadiamento clínico e o local de residência da mulher, sendo que mulheres residentes na capital apresentaram maior freqüência de diagnóstico do CM nos estádios I e II do que as mulheres residentes no interior do estado, conforme apresentado na Tabela 1.

Tabela 1- Distribuição do método de deteç̧ão e estadiamento clínico do câncer de mama

\begin{tabular}{lcccccc}
\hline \multicolumn{1}{c}{$\begin{array}{c}\text { Método de } \\
\text { Detecção* }\end{array}$} & I & II & III & IV & Total & \multirow{2}{*}{ p-valor } \\
\hline AEM & $15(8,8 \%)$ & $77(45,0 \%)$ & $76(44,4 \%)$ & $3(1,8 \%)$ & $171(100,0 \%)$ & 0,0001 \\
ECM & $10(29,4 \%)$ & $10(29,4 \%)$ & $13(38,2 \%)$ & $1(2,9 \%)$ & $34(100,0 \%)$ & 0,06 \\
Mamografia & $8(47,1 \%)$ & $4(23,5 \%)$ & $5(29,4 \%)$ & $0(0,0 \%)$ & $17(100,0 \%)$ & 0,002 \\
\hline
\end{tabular}

* Informação sobre estadiamento clínico não disponível para uma mulher

O estadiamento clínico esteve associado à forma de deteç̧ão do CM, conforme dados da Tabela 2.

Tabela 2 - Distribuição do estadiamento clínico do câncer de mama, segundo local de procedência

\begin{tabular}{lccccc}
\hline \multicolumn{5}{c}{ Estadiamento Clínico } \\
\hline Procedência & I & II & III & IV & $\begin{array}{c}\text { p- } \\
\text { valor }\end{array}$ \\
Capital & 27 & 64 & 54 & 2 & 0,0464 \\
& $(81,8 \%)$ & $(70,3 \%)$ & $(57,4 \%)$ & $(50,0 \%)$ & \\
Interior & 6 & 27 & 40 & 2 & \\
& $(18,2 \%)$ & $(29,7 \%)$ & $(42,6 \%)$ & $(50,0 \%)$ & \\
Total & 33 & 91 & 94 & 4 & \\
& 100,0 & 100,0 & 100,0 & 100,0 & \\
\hline
\end{tabular}

\section{DISCUSSÃO}

O CM apresenta-se como um grave problema de saúde pública em todo o mundo. Nas últimas décadas, o desenvolvimento da doença tem sido associado a diversos fatores de risco. Em países nos quais os recursos para a implementação de programas de rastreamento e detecção precoce são escassos, o conhecimento dos principais fatores de risco pode assumir especial papel na aplicação de estratégias que minimizem tais fatores ${ }^{(19)}$.

Com exceção do sexo feminino, a idade é o fator de risco mais importante, o risco do desenvolvimento do CM aumenta com a idade, 77\% dos casos novos da doença diagnosticados nos Estados 
Unidos entre os anos de 1994 e 1998, ocorreram em mulheres com idade igual ou superior a $50 \mathrm{anos}^{(20)}$.

No presente estudo a idade média das mulheres estudadas foi superior a 50 anos de idade, confirmando os achados encontrados por outros autores ${ }^{(8,21-22)}$. O $\mathrm{CM}$ é pouco comum em mulheres com idade inferior a 40 anos, constituindo aproximadamente $07 \%$ de todos os casos diagnosticados ${ }^{(23)}$. Embora a maior incidência do CM seja observada em mulheres com idades mais avançadas $^{(22)}$, as mulheres jovens, com idades inferior a 35 anos de idade apresentam piores prognósticos da doença ${ }^{(6)}$. Dessa forma, o planejamento de ações de rastreamento e detecção precoce deve considerar, portanto, a necessidade de incluir não só as mulheres com idade superior a 50 anos, mas também mulheres mais jovens, possibilitando também neste grupo a detecção do CM em estágios mais precoces.

Outro fator de risco para a doença é a história familiar, especialmente em primeiro grau. O papel dos antecedentes familiares na gênese do CM parece estar relacionado a fatores genéticos, como as mutações nos genes BRCA1 e BRCA2(2). Embora a história familiar seja um critério preponderante na definição dos grupos populacionais com risco elevado para o desenvolvimento do $\mathrm{CM}^{(17)}$, deve-se ressaltar que a prevalência deste fator de risco é baixa, encontrando-se entre 3,5 a 6,5\% em estudos anteriores ${ }^{(2,24)}$. Entre as mulheres incluídas neste estudo, encontrou-se prevalência de história familiar muito superior à relatada em outros estudos, isto possivelmente se deve ao fato de que, neste, considerou-se parentes de primeiro e segundo grau conjuntamente. Em estudo anterior ${ }^{(25)}$ em que foi verificada a associação de história familiar em parentes de segundo grau com o CM, a prevalência encontrada foi de $15,15 \%$, aproximando-se mais dos achados do presente estudo.

Vale ressaltar que o crescimento da taxa de incidência do CM observado nos últimos anos pode estar relacionado a fatores como a melhoria na qualidade dos registros ${ }^{(2)}$, a maior difusão de informações sobre os fatores de risco e métodos de detecção e o desenvolvimento de programas de rastreamento e deteç̧ão precoce.

Os Programas de rastreamento e detecção precoce do CM são, em maioria, realizados por três métodos, sendo eles: o AEM que apresenta baixo custo, alta acessibilidade, mas requer dedicação e treinamento da própria mulher para aumentar sua baixa eficácia; o ECM que apresenta custo baixo a moderado, nem sempre é acessível, depende da habilidade do clínico e sua eficácia é considerada moderada; e a mamografia, considerada o método mais eficaz na detecção de tumores iniciais da mama, possui custo moderado a alto e ainda é pouco acessível para grande parte das mulheres brasileiras ${ }^{(26)}$.

O método de detecção do tumor assume importante papel no que diz respeito ao seu tamanho no momento do diagnóstico. O estadiamento clínico TNM proposto pela União Internacional Contra o Câncer (UICC) considera o tamanho do tumor como uma das características preditoras do prognóstico da doença, dessa forma, tumores de menor diâmetro estão invariavelmente relacionados a um prognóstico mais favorável e maiores taxas de sobrevida ${ }^{(6)}$.

Entre os métodos de detecção do CM mais utilizados a mamografia é, sem dúvida, o que apresenta maior eficácia na detecção de tumores em estádios iniciais. Está bem estabelecido que o CM quando detectado durante rastreamento mamográfico apresenta menor estadiamento clínico se comparado aos tumores detectados pelos demais métodos, ou seja, são tumores menores, que apresentam menor disseminação em linfonodos axilares e possibilitam a realização de cirurgias conservadoras sem a necessidade de tratamentos de alta toxidade como a quimioterapia ${ }^{(16)}$.

Os achados no presente estudo demonstram que a grande maioria dos tumores ainda é detectada pela própria mulher, e apenas uma parcela restrita da população teve o CM detectado pela mamografia. No Brasil, aproximadamente $50 \%$ das mulheres com indicação do exame mamográfico, não conseguem realizá-lo pelo Sistema Único de Saúde (SUS) ${ }^{(27)}$ o que, na maioria dos casos, significa que o exame não será realizado devido a obstáculo econômico ou será realizado após longo período de espera, retardando o diagnóstico da doença e reduzindo as chances de cura.

Observou-se no presente estudo que o método de detecção do tumor mamário está significativamente associado ao estadiamento clínico, de forma que 47,1\% dos tumores detectados em estádio I foram detectados por meio do exame mamográfico, enquanto que o AEM e o ECM foram os métodos responsáveis pela detecção de $8,8 \%$ e $29,4 \%$ dos tumores no mesmo estádio, respectivamente.

Mesmo sendo considerado o método diagnóstico mais adequado, a mamografia apresenta-se como um exame radiológico de alto custo e, infelizmente, ainda hoje não está acessível a toda a população, principalmente entre mulheres de menor poder sócio-econômico(28). 
A utilização da mamografia também é limitada pela infra-estrutura (mamógrafos insuficientes), precariedade de controle da qualidade das imagens e engajamento do médico e da mulher ${ }^{(10)}$.

No Brasil, o diagnóstico do CM é em maioria realizado tardiamente ${ }^{(6)} \mathrm{o}$ que pode, entre outras coisas, refletir a ausência de uma política que propicie o cuidado adequado no que se refere ao CM. O diagnóstico tardio do CM implica em prejuízos para a mulher, já que as chances de cura da doença ficam reduzidas, ainda que sendo submetida a tratamentos mutilantes e, conseqüentemente, experimentar maior grau de sofrimento, repercutindo em todo o núcleo familiar. A saúde pública também sofre prejuízos, já que o sistema de saúde arca com gastos excessivos para o tratamento dessas mulheres, que poderiam ter recebido um diagnóstico precoce do $\mathrm{CM}^{(30)}$.

Em seu estudo ${ }^{(31)}$, comparando os custos do tratamento de mulheres com câncer de mama em estádio inicial e tardio, descrevem que o custo do tratamento dos casos detectados em estádio 0 e I varia em torno de $\mathrm{R} \$ 6.000,00$. Esse valor salta para R\$ $25.000,00$ a $\mathrm{R} \$ 62.500,00$ quando o tumor é detectado em estádios III e IV, assim, estima-se que com o valor destinado ao tratamento de duas a quatro mulheres com CM avançado, poderiam ser rastreadas 1000 mulheres, aumentando-se as chances de diagnóstico precoce.

Sabe-se que a implementação de um programa efetivo de rastreamento e detecção precoce do CM esbarra em diversas dificuldades, como a quantidade insuficiente e localização de mamógrafos, a precariedade de controle da qualidade das imagens e o engajamento do médico e da mulher para a realização do exame ${ }^{(10)}$.

Em relação à rede instalada de mamógrafos, a maioria dos aparelhos encontra-se na capital do Estado, sendo ainda poucas as cidades do interior que oportunizam o acesso à mamografia, entretanto, não houve diferença significativa entre os métodos de detecção utilizados e as diferentes procedências das mulheres estudadas, possivelmente pela dificuldade de acesso também na capital, a despeito do número de aparelhos.

Diferentemente, observou-se associação significativa entre a procedência da mulher e o estadiamento clínico, evidenciando-se que o número de diagnósticos realizados em estádio I, foi superior no grupo de mulheres residentes na capital do Estado. Isso certamente se deve ao fato da existência de uma rede de atenção à saúde mais ampla e pulverizada, oportunizando às mulheres maiores possibilidades de acesso aos cuidados de saúde, em especial ao ECM e às ações de educação em saúde.

\section{CONCLUSÃO}

A deteç̧ão do CM na amostra estudada foi em maioria realizada pela própria mulher, sendo que apenas uma pequena parcela de mulheres teve o tumor detectado por meio do exame mamográfico. O estadiamento clínico esteve associado ao método de detecção do tumor e também ao local de residência das mulheres, de forma que maior freqüência de diagnósticos precoces foi observada em mulheres que tiveram o tumor detectado pela mamografia e residentes na capital do Estado.

O planejamento de políticas voltadas à saúde da mulher deve, entre outros aspectos, minimizar as barreiras para que as mulheres tenham acesso à estratégias efetivas de rastreamento e detecção precoce do $\mathrm{CM}$, resultando em ganhos para a mulher, para o estado e para a sociedade.

\section{REFERÊNCIAS}

1. Instituto Nacional do Câncer (INCA). Estimativas 2008: Incidência de Câncer no Brasil[Internet]. Brasília (DF): Ministério da Saúde; 2007. [acesso em 2008 Set 10]. Disponível: http://www.inca.gov.br/estimativa/2008/

2. Pinho VFS, Coutinho ESF. Variáveis associadas ao câncer de mama em usuárias de unidades básicas de saúde. Cad Saúde Publ. 2007; 23(5):1061-9.

3. Pereira MG. Epidemiologia: teoria e prática. Rio de Janeiro: Guanabara Koogan; 1995.

4. Marinho LAB, Costa-Gurgel MS, Cecatti JG, Osis MJD. O papel do auto-exame mamário e da mamografia no diagnóstico precoce do câncer de mama. Rev Cienc Med. 2002; 11(3):233-42.

5. Molina L, Dalben I, De Luca LA. Análise das oportunidades de diagnóstico precoce para as neoplasias malignas de mama. Rev Assoc Med Bras. 1003; 49(2):185-90.

6. Abreu E, Koifman S. Fatores prognósticos no câncer de mama feminina. Rev Bras Cancerol. 2002; 48(1):113-31.

7. Paulinelli RR, Freitas Júnior R, Curado MP, Souza AA.A situação do câncer de mama em Goiás, no Brasil e no mundo: tendências atuais para a incidência e mortalidade. Rev Bras Saúde Matern Infant. 2003; 3(1):17-24. 
8. Menke $\mathrm{CH}$, Pohlmann PR, Backes A, Cericatto R, Oliveira M, Bittelbrunn A, Schwartsmann G. Tumor size as a surrogate end point for the detection of early breast cancer: a 30-Year (1972-2002), single-center experience in southern Brazil. Breast J. 2007; 13(5):448-56.

9. Feig SA. Screening mammography: a successful public health initiative. Rev Panam Salud Publica. 2006; 20(2/ 3):125-33.

10. Godinho ER, Koch HA. Rastreamento do câncer de mama: aspectos relacionados ao médico. Radiol Bras. 2004; 37(2):91-9.

11. Simon CE. Breast cancer screening: cultural beliefs and diverse populations. Health \& Social Work. 2006; 31(1):37-43.

12. Bancej C, Decker K, Chiarelli A, Harrisom M, Turner D, Brisson J. Contribution of clinical breast examination to mammography screening in the early detection of breast cancer. J Med Screen. 2003; 10(1):1-13.

13. Elmore JG, Armstrong K, Lehman CD, Fletcher SW. Screening for breast cancer. JAMA. 2005; 293(1):1245-56.

14. Kudadjic-Gyamfi E, Consedine N, Magai C, Gillespie M, Pierre-Louis J. Breast self-examination practices among women from six ethnic groups and the influence of cancer worry. Breast Cancer Res Treat. 2005; 92:35-45.

15. Shen Y, Yang Y, Inoue LYT, Munsell MF, Miller AB, Berry DA. Role of detection method in predicting breast cancer survival: analysis of randomized screening trials. J Natl Cancer Inst. 2005; 97(13):1195-203.

16. Chapgar AB, McMasters KM. Trends in mammography and clinical breast examination: a population-based study. J Surg Res. 2007; 140: 214-19.

17. Instituto Nacional do Câncer (INCA).Controle do câncer de mama: documento de consenso [Internet]. Brasília (DF): Ministério da Saúde; 2004 [acesso em 2008 Mar 18]. Disponível em: http://www.inca.gov.br/publicacoes/ Consensointegra.pdf.

18. Organização Mundial da Saúde. Classificação estatística internacional de doenças e problemas relacionados à saúde. São Paulo: EDUSP; 1994.

19. Musre OP. Cáncer de Mama. Epidemiología y factores de riesgo. Cad Méd Soc (Chile) 2007; 47(1):18-30.

20. American Cancer Society. Breast Cancer, Facts \& Figures2001-2002 [Internet].[acesso em 2008 Mar 18]. Disponível: http://www.cancer.org/downloads/STT/ BrCaFF2001.pdf.
21. Trufelli DC, Miranda VC, Santos MBB dos, Fraile NMP, Pecoroni PG, Gonzaga SFR et al . Análise do atraso no diagnóstico e tratamento do câncer de mama em um hospital público. Rev Assoc Med Bras 2008; 54(1):72-6.

22. Paiva CE, Ribeiro BS, Godinho AA, Meirelles RSP,Silva EVG da, Marques GD, Rossini Júnior O. Fatores de Risco para Câncer de Mama em Juiz de Fora (MG): um estudo caso-controle. Rev Bras Cancerol. 2002; 48(2):231-7.

23. Winchester DP. Breast cancer in young women. Surg Clin North Am. 1996; 76(2):279-87.

24. Lima JQ, Falk JA. A importância da identificação dos fatores de risco na luta contra o câncer. $2^{\mathrm{a}}$ ed. Recife: Sociedade Pernambucana de Combate ao Câncer; 1998.

25. Souza RM de, Lazzaron AR, Defferrari R, Borba AA, Scherer L, Frasson AL. História familiar em segundo grau como fator de risco para câncer de mama. RBGO. 1998; 20(8):469-73.

26. Aguillar VLN, Bauab SP. Rastreamento mamográfico para detecção precoce do câncer de mama. Rev Bras Mastol. 2003; 13(2):82-9.

27. Cellis JAR. Avances en el diagnóstico del cáncer de la mama. Importancia de la pesquisa y diagnóstico precoz. Reflexiones sobre el problema en Venezuela. Gac Méd Caracas. 2001; 109(3):389-417.

28. Dias-da-Costa JS, Olinto MTA, Bassani D, Marchionatti CRE, Bairros FS, Oliveira MLP, Hartmann M, Gabardo RM, Bennemann V, Pattussi MP. Desigualdades na realização do exame clínico de mama em São Leopoldo, Rio Grande do Sul, Brasil. Cad Saúde Publ. 2007; 23(7):1603-12.

29. Brasil. Ministério da Saúde. Instituto Nacional de Câncer. A situação do câncer no Brasil. 2006 [Internet]. [acesso em 2008 Mar 18]. Disponível: www.inca.gov.br/ situacao/arquivos.pdf.

30. Marinho LAB, Costa- Gurgel MS, Cecatti JG, Osis MJD. Conhecimento, atitude e prática do autoexame das mamas em centros de saúde. Rev Saúde Publ. 2003; 37(5):576-82.

31. Kemp C, Elias S, Gebrim LH, Nazário ACP, Baracat EC, Lima GR. Estimativa de custo do rastreamento mamográfico em mulheres no climatério. Rev Bras Ginecol Obstet. 2005; 27(7):415-20. 